\title{
Treatment compliance and therapy satisfaction in HIV-infected patients
}

\author{
Ewa Siwak ${ }^{1,2}$, Małgorzata Lemańska ${ }^{3}$, Ewa Firląg-Burkacka ${ }^{1,2}$, Wojciech Tomczyński ${ }^{4}$, Mariusz Baj ${ }^{4}$, \\ Maria Jankowska ${ }^{3}$ \\ ${ }^{1}$ HIV Out-Patient Clinic in Warsaw, Poland \\ ${ }^{2}$ Hospital of Infectious Diseases in Warsaw, Poland \\ ${ }^{3}$ Department of Infectious Diseases, Medical Univerisity of Gdansk, Poland \\ ${ }^{4}$ Polish National Network of PLWHA "SIEC PLUS"
}

\begin{abstract}
Introduction: Adherence to the prescribed therapy is the most important factor influencing the treatment outcome. The aim of study was to assess human immunodeficiency virus (HIV) patients' compliance and satisfaction with recommended antiretroviral and concomitant therapies.

Material and methods: A survey was conducted among HIV-infected patients. Therapeutic regimens for HIV and co-morbidities were described by the number of daily doses and total daily number of tablets. Patients' satisfaction was assessed on a six-point scale, and a Visual Analogue Scale (VAS) was used to assess the compliance with a recommended therapy.

Results: A total of 120 patients $(M=87, F=33)$ aged 18-72 (46 \pm 15$)$ years with HIV treated for 1-288 $(109 \pm 77)$ months filled in the questionnaire. Patients received 1-9 tablets in 1-3 doses a day as part of the antiretroviral therapy $-36 \%$ of subjects received only one tablet a day, and $74 \%$ received treatment in one daily dose. Eighty-nine percent of subjects were 'very satisfied' and 'satisfied' with the received anti-HIV treatment. Therapeutic compliance was the best for patients receiving only one tablet per day as single-tablet regimens (STR). Eighty patients also received 1-21 tablets in 1-> 3 daily doses of co-morbidities therapy. Fifty-four percent of patients received non-HIV therapy in one single dose. Eighty-one percent of subjects were 'very satisfied' and 'satisfied' with treatment for other-than-HIV diseases.

Conclusions: In the population under study, patients' compliance and satisfaction with both HIV and non-HIV concomitant disease therapies were high. The compliance with recommended HIV therapy was the best for patients receiving only one tablet per day, and the satisfaction with antiretroviral treatment was greater in patients receiving $\leq 2$ tablets per day.
\end{abstract}

HIV AIDS Rev 2018; 17, 3: 164-168 DOI: https://doi.org/10.5114/hivar.2018.78486

Key words: HIV, treatment compliance, treatment satisfaction.

\section{Introduction}

The progress in medicine has improved long-term prognoses with respect to many diseases. On a frequent basis, new medications emerge characterised with ever-improving effectiveness and fewer adverse events. However, some therapies remain complex when it comes to the number of daily doses of tablets that a patient is required to take, which, at

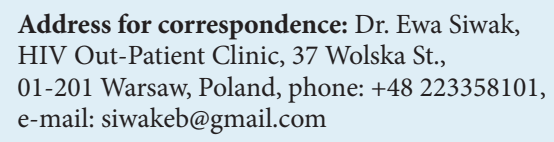

Article history:

Received: 25.01.2018

Received in revised form: 15.04.2018

Accepted: 19.04.2018

Available online: 15.08 .2018
International Journal of HIV-Related Problems

HIV \& AIDS

R e vi e w 
times, makes the treatment regimen difficult to follow. It is therefore anticipated that a patient's non-compliance with a recommended treatment schedule has a negative impact on the efficacy of the therapy.

The problem of non-compliance with the prescribed treatment schedule is well recognised. It exists in all therapeutic areas and is especially important in chronic diseases requiring long-term treatment. There are several factors that increase the chance that the patient will not adhere to the prescribed therapeutic regimen: the therapy may be too complicated (i.e. daily number of tablets and dosing frequency); side-effects may be present or a risk factor, or they may not be acceptable to a patient; one cannot afford the financial costs of the therapy; or, finally, there may be several psychological reasons that cause a patient to stop treatment.

Human immunodeficiency virus (HIV) infection is an example of the chronic disease that needs constant, longterm therapy with antiretroviral medications. Treatment adherence is regarded as an important factor in achieving optimum outcomes in the treatment of HIV [1]. Poor adherence to antiretroviral therapy (ART) is associated with less effective viral suppression, which risks the health of the patient, and may also lead to permanent treatment resistance to that particular agent or a group of agents within a given combination therapy regimen. This may have detrimental effects on therapeutic options as well as treatment costs [2].

The current, effective antiretroviral therapy allows HIV patients to have life expectancy similar to that of the general population $[3,4]$. These patients thus become susceptible to age-related diseases such as cardiovascular diseases, diabetes, chronic kidney diseases, osteoporosis, and malignancies $[5,6]$. The presence of co-morbidities can increase the number of prescribed medications and negatively influence the compliance with a recommended therapy for HIV and other diseases.

The aim of the study was to assess the compliance with different regimens of antiretroviral therapy and to assess patients' satisfaction with those regimens. Additionally, we asked our patients to assess the compliance and satisfaction with the therapy for other-than-HIV concomitant diseases.

\section{Material and methods}

A survey was conducted among patients with HIV randomly selected from attendees to the educational event organised by "Sieć Plus" (non-governmental organisation working for HIV-infected patients) in 2015. The questionnaire identified patients' key demographic, the history of HIV infection, and the presence of other-than-HIV concomitant diseases. Patients were asked to describe only their current treatment regimen (regardless of previous therapies and treatment failures) defined as 'the number of doses and the total number of tablets taken every day for the therapy of HIV and other co-morbidities.

The compliance with prescribed therapy for HIV or for other medical conditions was assessed with a Visual Analogue Scale (VAS). Patients were asked to rate their satisfac- tion with the therapy using a six-point scale (very satisfied/ satisfied/rather satisfied/difficult to say/unsatisfied/very unsatisfied).

Patients' satisfaction and compliance with prescribed medication for HIV and other-than-HIV medical conditions were compared with the number of daily tablets.

Descriptive statistics methods were used for the presentation of patients' demographic profiles and treatments. Non-parametric statistical tests (Fisher exact and Mann-Whitney) were used to compare patients' satisfaction with the treatment regimen (number of tablets). The Spearman test was used to correlate compliance with ART and other-than-HIV therapies.

Patients' participation in the survey was voluntary and did not influence subsequent medical care or medical decisions. The collected data was analysed anonymously using epidemiological methodology, so the study protocol did not require approval from the Ethics Committee.

\section{Results}

A total of 120 patients $(\mathrm{M}=87, \mathrm{~F}=33$ ) aged 18-72 (mean \pm SD: $46 \pm 15$ ) years filled in the questionnaire. Patients had been infected with HIV for 1-348 (146 \pm 103$)$ months and had been receiving antiretroviral therapy for 1-288 (109 \pm 77$)$ months.

The most frequent concomitant diseases were arterial hypertension ( $n=35.29 \%)$, hyperlipidaemia $(n=30,25 \%)$, hepatotropic viruses infections (HBV: $n=8,7 \%$; HCV: $n=25$, $21 \%$; HBV+HCV: $n=6,5 \%)$ and ischaemic heart disease $(n=7,6 \%)$. The other medical diagnoses were present in less than $5 \%$ of the studied population.

\section{HIV treatment regimen}

The number of daily doses of anti-HIV medication varied from one to three, and the total daily number of anti-HIV tablets from one to nine (Table 1 ). The majority of subjects $(n=88,74 \%)$ received anti-HIV medications in one daily dose. More than half of the patients received fewer than three anti-HIV tablets a day (Table 1).

Patients assessed their compliance with prescribed antiHIV medications to be between 15 and 100\% (93 \pm 17 ). Median compliance with anti-HIV medication was 100\% (Q1-Q3: 95-100). There were no statistical differences between men and women in compliance with ARV therapy.

The majority of patients ( $n=104,89 \%)$ were 'satisfied' or 'very satisfied' with their current anti-HIV regimens (Table 2). Patients who were on one- or two-tablet regimens showed a statistically higher $(p=0.011)$ satisfaction with the therapy than those receiving more than two tablets (Figure 1). Compliance with a prescribed therapy was statistically higher in patients treated with one tablet per day (Mann-Whitney $p=0.043$ ). There were no statistical differences between men and women in the satisfaction with antiretroviral treatment. 

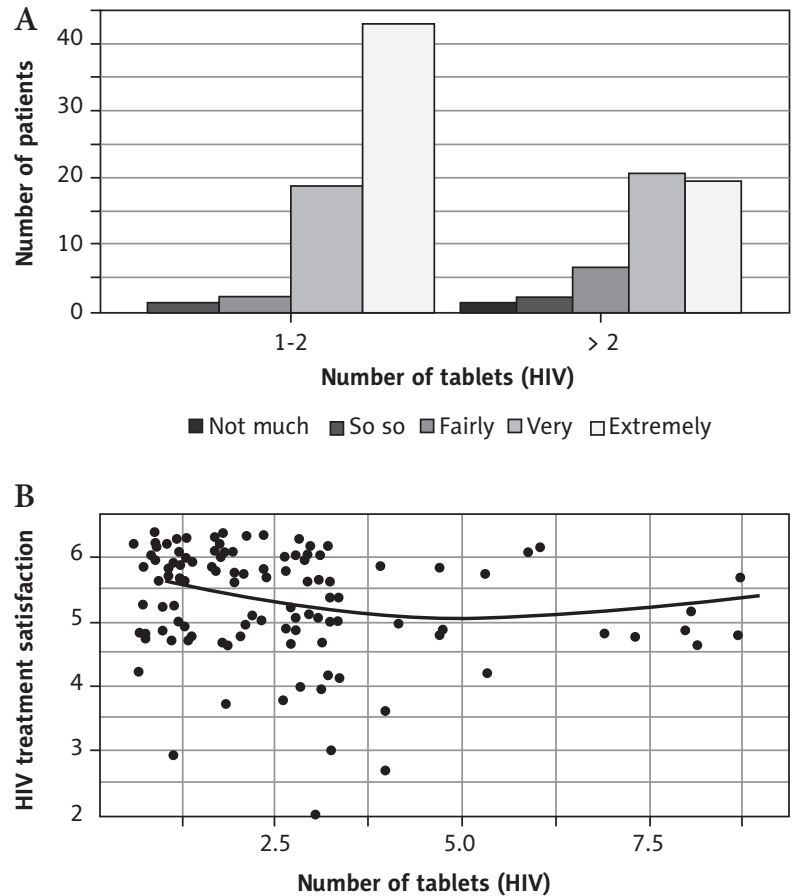

Fig. 1. Patients' satisfaction with 'no more than 2' vs. 'more than 2' tablet regimens

\section{Concomitant diseases treatment regimen}

A total of 80 patients (67\%) were treated also for co-morbidities. The number of daily doses of medications prescribed for other-than-HIV diseases varied from 1 to $>3$ and the total daily number of tablets from 1 to 21 (Table 3 ). Over half of the subjects (54\%) received medications in one daily dose, and over half of patients (51\%) received more than two tablets a day for concomitant diseases.

Patients assessed their compliance with medication administered for other-than-HIV concomitant diseases to be between 0 and $100 \%(89 \pm 26)$. Median compliance was $100 \%$ (Q1-Q3: 95-100).

The majority of patients (81\%) were 'satisfied' or 'very satisfied' with the treatment regimen recommended for concomitant disease (Table 2). Patients's satisfaction was not related to the total daily number of tablets (Fisher $p=0.536$ ). The compliance with a prescribed therapy in patients receiving only one tablet per day was not higher than in those receiving more tablets (Mann-Whitney $p=0.228$ ).

\section{Comparison of adherence to HIV therapy with adherence to concomitant disease treatment}

Our data showed a positive correlation between adherence to HIV therapy and adherence to concomitant diseases treatment (Sperman test $r-0.535 ; p<0.001$ ) (Figure 2).
Table 1. Anti-HIV treatment regimen: number of daily doses and total daily number of tablets

\begin{tabular}{l|c|c|c}
\hline \multicolumn{2}{l|}{ Daily anti-HIV treatment regime } & $n$ & $\%$ \\
\hline \multirow{4}{*}{ Number of doses } & 1 & 88 & 74 \\
\cline { 2 - 4 } & 2 & 28 & 24 \\
\cline { 2 - 4 } & 3 & 2 & 2 \\
\hline \multirow{4}{*}{ Total number of tablets } & 1 & 43 & 36 \\
\cline { 2 - 4 } & 2 & 23 & 19 \\
\cline { 2 - 4 } & 3 & 34 & 29 \\
\cline { 2 - 4 } & $>3$ & 18 & 16 \\
\hline
\end{tabular}

Table 2. Patients' satisfaction with the current treatment regimen for HIV and other-than-HIV concomitant diseases

\begin{tabular}{l|c|c|c|c}
\hline \multirow{2}{*}{} & \multicolumn{2}{|c|}{ Anti-HIV therapy } & \multicolumn{2}{c}{$\begin{array}{c}\text { Concomitant } \\
\text { diseases therapy }\end{array}$} \\
\cline { 2 - 5 } & $n$ & $\%$ & $n$ & $\%$ \\
\hline Very satisfied & 64 & 55 & 25 & 34 \\
\hline Satisfied & 40 & 34 & 35 & 47 \\
\hline Rather satisfied & 9 & 8 & 9 & 12 \\
\hline Difficult to say & 3 & 2 & 5 & 7 \\
\hline Not satisfied & 1 & 1 & 0 & 0 \\
\hline Very unsatisfied & 0 & 0 & 0 & 0 \\
\hline
\end{tabular}

Table 3. Concomitant diseases treatment regimen: number of daily doses and total daily number of tablets

\begin{tabular}{l|c|c|c}
\hline \multicolumn{2}{l|}{$\begin{array}{l}\text { Daily treatment regime for other } \\
\text { than HIV diseases }\end{array}$} & $n$ & $\%$ \\
\hline \multirow{4}{*}{ Number of doses } & 1 & 43 & 54 \\
\cline { 2 - 4 } & 2 & 27 & 33 \\
\cline { 2 - 4 } & 3 & 7 & 9 \\
\cline { 2 - 4 } & $>3$ & 3 & 4 \\
\hline \multirow{4}{*}{ Total number of tablets } & 1 & 21 & 26 \\
\cline { 2 - 4 } & 2 & 18 & 23 \\
\cline { 2 - 4 } & 3 & 18 & 23 \\
\cline { 2 - 4 } & $>3$ & 23 & 28 \\
\hline
\end{tabular}

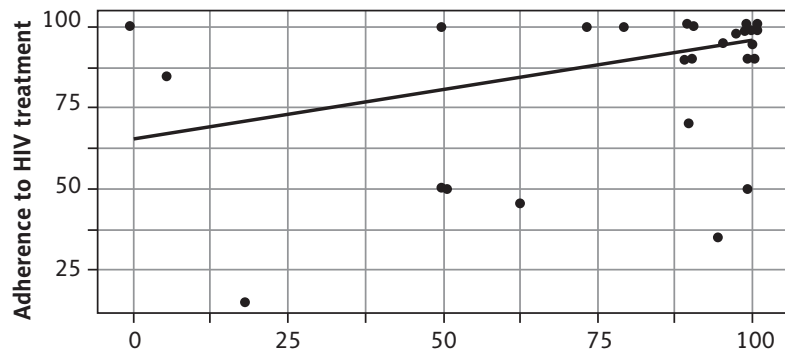

Adherence to treatment of concomitant diseases

Fig. 2. Correlation between adherence to treatment of HIV and concomitant diseases 


\section{Discussion}

The problem of compliance with the prescribed antiretroviral treatment regimen is widely discussed in the recent literature. Because it is particularly important in countries with a high prevalence of HIV infection, many research papers on that topic come from Africa and Asia [7, 8].

The number of patients in the majority of the reports discussing the compliance with ART is similar to that described in this paper. The population described in this paper is slightly older (median age 46 years) and the duration of ART (median nine years) is longer as compared to that described by other Polish authors [9]. This is related to a specific location of our site and may be the reason why the results observed in our group differ from other reports. We can speculate that patients who have been living with a disease for longer are better educated regarding the disease and their degree of disease understanding is higher than that in younger patients with more recent infection. It was shown that behavioural interventions (including education about antiretroviral medication and their side effects), positive reinforcement and encouragement, lifestyle assessment, and the identification of adherence barriers could markedly increase adherence to treatment [10]. Some authors recommend including patient's self-reporting of adherence together with routine measurements of viral load in routine practice [11]. The combined use of information and communication technologies and standard care promotes increased access to care, strengthening of the relationship between patients and health services, and can improve patients' compliance with a recommended therapy [12].

Treatment compliance may be measured using different methodologies. The simplest way is the evaluation of the number of tablets taken daily. More advanced approaches include self-reported questionnaires and pharmacologic assays of drug levels in randomly-drawn blood samples, but each of these has important limitations [13, 14]. Wójcik et al. [9], using the Morisky eight-item Medical Adherence Questionnaire, showed high adherence in only $41 \%$ of the subjects, while $19 \%$ of patients had low adherence. In this study, we used patients' self-assessment questionnaire scores together with the analogue scale scores for compliance assessment. This approach is not controlled by any objective factors, and the results may be biased.

One of the controlling factors for treatment adherence may be the efficacy of treatment. This aspect was not analysed in our study, but Wójcik et al. [9] showed that patients with better compliance had better virological effect. The cross-sectional study of 5152 Polish HIV-infected patients (56.9\% of the country-wide treated patients on ART) demonstrated that the antiretroviral treatment success defined as 'undetectable HIV (viral load < 50 copies/ml)' was achieved in over $90 \%$ of the subjects [15]. These findings may confirm the very high compliance rate (median 100\%) declared by the patients in our study. A recent meta-analysis [16] of 43 studies including over 27,000 subjects confirmed that the adherence level influences the virological outcome. The authors stated that the threshold for optimum adherence to achieve better virological response appeared to be wider than the commonly used $\geq 95 \%$. They concluded that patients on antiretroviral therapy should be instructed to attain at least $95 \%$ adherence.

Another meta-analysis [17] of 36 studies including almost 7000 Chinese subjects showed remarkable variations in adherence rates in different populations, with lower adherence in: patients from heterosexual transmission group, former blood or plasma donors, and intravenous drug users, and high level of adherence in: older patients (as in our study), children, and pregnant women. It is recommended that comprehensive intervention strategies be developed to promote ART adherence in high-risk populations and effectively protect against the spread of HIV/AIDS.

The complexity of treatment regimens influences compliance [1]. Sebally and Kelley showed that a single-tablet regimen markedly improved patients' compliance [18]. The same observation was made by Langness et al. [19]. In our study, the majority of subjects received one- or two-pill regimens (56\%). Almost $75 \%$ of the subjects received their medication in one daily dose. This may be the reason why patients reported very high compliance rates and that they seemed to be very satisfied with recommended ART. Our data show that patients who receive one or two tablets a day are more satisfied than those receiving three or more tablets.

It is interesting that, in the case of comorbidities, patients are not paying so much attention to the number of pills and doses, in contrast to HIV therapy. We can speculate that HIV-infected patients attach greater importance to the quality of antiretroviral treatment as their most important treatment, which saves their lives, rather than treating other diseases. On the other hand, the place and time where patients completed survey could be relevant - educational meeting for patients where HIV problems were mainly discussed.

Another aspect of our study is the comparison of compliance with ART with the compliance with other-than-HIV concomitant diseases. Many studies have shown that among people with chronic illness, people living with HIV are most likely to follow recommendations for taking medications. Our data show that, in both cases, compliance with antiretroviral therapy and other co-comorbidities are similar and high. Galan et al. and Lemon et al. stated that the presence of concomitant disease might decrease compliance with ART $[20,21]$. Other authors showed better adherence to antiretroviral treatment than for other comorbidities such as diabetes [22], hypertension, or mental illness in HIV-infected patients [19]. The observations and meta-analysis of supervised research imply that compliance grows when the patient is motivated at every visit, educated, and controlled by the number of taken medications. The Polish National HIV/AIDS Treatment and Prevention Program obliges patients to attend visits on the outpatient clinic every 1-3 months; then they receive prescriptions for antiretroviral medications (free of charge). Such a system allows for patients' increased motivation, education, and control of drug use, which ultimately makes the patient more likely to adhere to the therapy. 


\section{Conclusions}

The data presented in this review comes from everyday practice of the HIV Outpatient's Clinic. In the studied population, both patients' compliance and satisfaction with HIV and non-HIV-concomitant disease therapies were high. The compliance with recommended HIV therapy was best for patients receiving only one tablet per day, and the satisfaction with antiretroviral treatment was greater in patients receiving one or two tablets per day.

\section{Acknowledgments}

The authors would like to thank GILEAD for their support, MANTEION LABORATORIUM STATYSTYCZNE for statistical support, and MEDICAL NETWORK for editorial support.

\section{Conflict of interest}

The authors declare no potential conflicts of interest with respect to the research, authorship, and/or publication of this article.

\section{References}

1. Ammassari A, Trotta MP, Murri R, et al. Correlates and predictors of adherence to highly active antiretroviral therapy: overview of published literature. J Acquir Immune Defic Syndr 2000; 31: 123-127.

2. Schaker KL. The importance of Treatment Adherence in HIV. Am J Manag Care 2013; 19 (12 Suppl): 231-237.

3. Aguiar C, Antela A, Compston J, et al. Going beyond undetectable: a review of the unmet long-term health needs of people living with HIV. HIV Therapy 2015; 1: 1-12.

4. Rasmussen LD, May MT, Kronborg G, et al. Time trends for risk of severe age-related diseases in individuals with and without HIV infection in Denmark: a nationwide population-based cohort study. Lancet HIV 2015; 2: e288.

5. Smit M, Brinkman K, Geerlings S, et al. Future challenges for clinical care of an ageing populationinfected with HIV: a modelling study. Lancet Infect Dis 2015; 15: 810-818.

6. Warrine AH, Greer A, Burkholder GA, Overton ET. HIV-Related Metabolic Comorbidities in the Current ART Era. Infect Dis Clin N Am 2014; 28: 457-476.

7. Kapiamba G, Masango T, Mphuthi D. Antiretroviral adherence and virological outcomes in HIV-positive patients in Ugu district, KwaZulu-Natal province. Afr J AIDS Res 2016; 15: 195-201.

8. Hasabi IS, Shivashankarappa AB, Kachapur C, Kaulgud RS. A Study of Compliance to Antiretroviral Therapy among HIV Infected $\mathrm{Pa}$ tients at a Tertiary Care Hospital in North Karnataka. J Clin Diagn Res 2016;10: 27-31.

9. Wójcik K, Piekarska A, Jabłonowska E. Adherence to Antiviral Therapy in HIV ir HBV Infected patients. Przegl Epidemiol 2016; 70: 27-32.

10. Molassiotis A, Lopez-Nahas V, Chung WY, Lam SW. A pilot study of the effects of a behavioural intervention on treatment adherence in HIV-infected patients. AIDS Care 2003; 15: 125-135.

11. Dagli-Hernandez C, Lucchetta RC, de Nadai TR, et al. Self-perception of knowledge and adherence reflecting the effectiveness of antiretroviral therapy. Patient Prefer Adherence 2016; 10: 1787-1793.

12. Lima IC, Galvão MT, Alexandre Hde O, et al. Information and communication technologies for adherence to antiretroviral treatment in adults with HIV/AIDS. Int J Med Inform 2016; 92: 54-61.
13. Kim HM, Lagakos SW. Assessing drug compliance using longitudinal marker data, with application to AIDS. Stat Med 1994; 13: 2141-2153.

14. Wilson IB, Lee Y, Michaud J, et al. Validation of a New Three-Item Self-Report Measure for Medication Adherence. AIDS Behav 2016; 20: 2700-2708.

15. Parczewski M, Siwak E, Leszczyszyn-Pynka M, et al. Meeting the WHO 90\% target: antiretroviral treatment efficacy in Poland is associated with baseline clinical patient characteristic. J Int AIDS Soc 2017; 20: 21847.

16. Bezabhe WM, Chalmers L, Bereznicki LR, Person GM. Adherence to Antiretroviral Therapy and Virologic Failure. A Meta-Analysis. Medicine (Baltimore) 2016; 95: e3361.

17. Huan Z, Fuzhi W, Lu L, et al. Comparison of Adherence to Antiretroviral Therapy in a High-Risk Population in China: A Systematic Review and Meta-Analysis. PLoS One 2016; 11: 20146659.

18. Sebaaly JC, Kelley D. Single-Tablet Regimens for the Treatment of HIV-1 Infection. Ann Pharmacother 2017; 51: 332-344.

19. Langness J, Cook PF, Gil J, et al. Comparison of adherence rates for antiretroviral, blood pressure, or mental health medications for hiv-positive patients at an academic medical center outpatient pharmacy. J Manag Care Spec Pharm 2014; 20: 809-814.

20. Jimenez Galan R, Montes Escalante IM, Morillo Verdugo R. Influence of pharmacotherapy complexity on compliance with the therapeutic objectives for HIV+ patients on ART concomitant with therapy for dyslipidemia. INCOFAR Project Farm Hosp 2016; 40: 90-101.

21. Lemos LA, Fiuza ML, Reis RK, et al. Adherence to antiretrovirals in people coinfected with human immunodeficiency virus and tuberculosis. Rev Lat Am Enfermagen 2016; 24: e2691.

22. Batchelder AW, Gonzalez JS, Berg KM. Differential medication nonadherence and illness beliefs in co-morbid HIV and type 2 diabetes. J Behav Med 2014; 37: 266-275. 